\title{
A intergeracionalidade entre idosos e adolescentes na busca da desconstrução de estereótipos na velhice: relato de experiência
}

Intergenerationality between the elderly and adolescents in the search for the deconstruction of stereotypes in old age: experience report

La intergeneracionalidad entre ancianos y adolescentes en la búsqueda de la deconstrucción de estereotipos en la vejez: relato de experiencia

Viviane Ferraz Ferreira de Aguiar ${ }^{1 *}$, Thainara Braga Soares ${ }^{1}$, Ana Flávia Silva dos Anjos ${ }^{1}$, João Victor Cunha Paz ${ }^{1}$, Jhemily Aires Amorim¹, Márcia Cristina Silva Torres ${ }^{1}$, Jailma Bendelaque Sousa1, Luane Freitas de Araújo ${ }^{1}$, Maria de Nazaré Gonçalves Pereira Ferreira1', Jully Gabriela Navegantes dos Santos ${ }^{1}$, Gabrielle Braga Rangel ${ }^{1}$.

\section{RESUMO}

Objetivo: Realizar uma ação educativa quanto à intergeracionalidade entre idoso e adolescente na busca da desconstrução de estereótipos na velhice. Detalhamento do caso: Foram realizadas quatro atividades, a saber: I- Dinâmica "Juntos e Misturados" com os adolescentes; II- Encontro entre os adolescentes e as idosas.; III- Dinâmica "Juntos e Misturados" na percepção das idosas; e IV- O balanço da roda entre duas gerações. Alguns participantes destacarem que foi a primeira vez que desenvolveram uma ação entre dois grupos e que ficaram satisfeitos. O principal desafio encontrado pelos acadêmicos na condução dessa ação foi o receio da não aceitação, contudo, o feedback e o resultado de cada etapa permitiram entender 0 fortalecimento da proposta de que mesmo com a singularidade de cada grupo é possível a intergeracionalidade. Considerações finais: A ação realizada permitiu identificar que a interação entre adolescentes e adultos é possível e que é uma estratégia que merece ser estudada na busca da desconstrução dos estereótipos da velhice.

Palavras-chave: Educação em Saúde, Idoso, Adolescente, Relação entre Gerações.

\begin{abstract}
Objective: To carry out an educational action regarding the intergenerationality between the elderly and the adolescent in the search for the deconstruction of stereotypes in old age. Case detail: Four activities were carried out, namely: I - Dynamics "Together and Mixed" with adolescents; II- Meeting between adolescents and the elderly.; III- Dynamics "Together and Mixed" in the perception of the elderly; and IV- The balance of the wheel between two generations. Some participants pointed out that this was the first time they had developed an action between two groups, and they were satisfied. The main challenge faced by the academics in the conduct of this action was the fear of not accepting, however, the feedback and the result of each stage allowed to understand the strengthening of the proposal that even with the uniqueness of each group it is possible to intergenerationality. Final considerations: The action carried out allowed us to identify that the interaction between adolescents and adults is possible and is a strategy that deserves to be studied in the search for the deconstruction of old age stereotypes.
\end{abstract}

Keywords: Health Education, Aged, Adolescent, Intergenerational Relations.

${ }^{1}$ Centro Universitário Metropolitano da Amazônia (UNIFAMAZ), Belém-Pará.

*E-mail: viviane.ferraz@yahoo.com.br

SUBMETIDO EM: 1/2019 


\section{RESUMEN}

Objetivo: Realizar una acción educativa cuanto a la intergeneracionalidad entre personas de edad avanzada y adolescente en la búsqueda de la desconstrucción de estereotipos en la vejez. Detalle del Caso: Fueron realizadas cuatro actividades, a saber: I - Dinámica "Juntos y mezclados" con los adolescentes: II- Encuentro entre los adolescentes y las personas de edad avanzada; III - Dinámica "Juntos y mezclados" en la percepción de las personas de edad avanzada; y IV- El balance de rueda entre dos generaciones. Algunos participantes destacaron que fue la primera vez que desarrollaron una acción entre dos grupos y que se quedaron satisfechos. El principal reto encontrado por los académicos en la conducción de esa acción fue el recelo de la no aceptación, pero, el feedback y el resultado de cada etapa permitió entender el fortalecimiento de la propuesta de que mismo con la singularidad de cada grupo es posible la intergeneracionalidad. Consideraciones finales: La acción realizada permitió identificar que la interacción entre adolescentes y adultos es posible y que es una estrategia que merece ser estudiada en la búsqueda de la deconstrucción de los estereotipos de la vejez.

Palabras clave: Educación en Salud, Anciano, Adolescente, Relaciones Intergeracionales.

\section{INTRODUÇÃO}

No Brasil, as projeções de idosos indicam que em 2050 a população brasileira será de 253 milhões de habitantes, a quinta maior população do mundo (MIRANDA G et al., 2016). Com o crescimento populacional e a alta expectativa de vida, medidas para a melhoria na qualidade de vida dos idosos são necessárias como a garantia de direitos, envelhecimento ativo e participação nos processos políticos e em outros aspectos da vida em comunidade (DÁTILO G e CORDEIRO A, 2015).

Contudo, observa-se que a população, considerada jovem, ainda possui uma visão sobre o idoso e a velhice enraizada em conceitos estereotipados. Torres TL et al. (2016) em sua pesquisa sobre o idoso identificou que os adolescentes destacaram alguns estereótipos negativos como ranzinzas, depressivos e ultrapassados. Tais denominações, ainda comum na sociedade, resultam na exclusão social.

Para Martínes Ques ÁA (2015) a exclusão social merece destaque em razão de aumentar gradativamente a presença de doenças e complicações para o idoso como isolamento, depressão e até mesmo o suicídio. Em razão destes aspectos negativos a discussão sobre o envelhecimento ativo se destaca, pois deve ser identificado como um processo de fortalecimento das oportunidades de saúde, participação e segurança (DAWALIBI NH et al., 2013).

Novas estratégias estão sendo desenvolvidas como a intergeracionalidade, visando a inclusão do idoso na sociedade atual. O conhecimento e as interações entre as diferentes gerações são essencialmente importantes para promover atitudes positivas sobre o outro. Dessa forma, ações que integram idosos, adultos, jovens, adolescentes e crianças tornam-se necessárias para que ocorra a desestruturação de estereótipos e preconceitos relacionados à idade (TARALLO RS et al., 2017).

A intergeracionalidade é definida como vínculos que se estabelecem entre duas ou mais pessoas com idades distintas, possibilitando o cruzamento de experiências e contribuindo para a harmonização dentro da diversificação. Ambas gerações podem ser beneficiadas a partir da percepção sobre a necessidade da interação, troca de suas singularidades no modo de ser, sentir, pensar e querer de cada um (SILVA DM et al., 2015).

As relações intergeracionais apresentam como principais objetivos a promoção da inclusão e valorização dos idosos, pois permite o compartilhamento de conhecimentos e valores humanos e estimula o mais jovem a ter um olhar diferente sobre os mais velhos, incentivando a aprendizagem por meio da educação informal (SEQUEIRA MS, 2014). Carmo AJCG (2017) acrescenta que é uma prática que permite manter as gerações mais próximas a partir de perspectivas diferentes de cada público-alvo. Ressalta que deveria ser uma ação inclusa nos sistemas de ensino para o desenvolvimento da prática de aprendizagem intergeracional. 
Neste contexto, o estudo justifica-se por haver a necessidade de se refletir sobre estratégias que permitam o vínculo entre faixas etárias diferentes. Portanto, o objetivo deste estudo foi realizar uma ação educativa quanto à intergeracionalidade entre idoso e adolescente na busca da desconstrução de estereótipos na velhice.

\section{DETALHAMENTO DO CASO}

Trata-se de um estudo descritivo, do tipo relato de experiência. Foi realizado por acadêmicos de enfermagem do 6o período, de uma Instituição Privada de Ensino, na cidade de Belém-Pará, no mês de outubro de 2018. A escolha da temática emergiu a partir das aulas de Enfermagem na Atenção à Saúde do Idoso. Para o conhecimento científico sobre o assunto de intergeracionalidade e o envelhecimento inicialmente foi realizado um levantamento bibliográfico. Com os achados encontrados a equipe se reuniu para a definição do problema e construção da metodologia a ser utilizada. Identificou-se como problema atual: "o aumento do número de idosos e sua exclusão pelos jovens que vivenciam uma era moderna e apresentam visão estereotipada do idoso".

O público alvo foi constituído por 26 adolescentes do $6^{\circ}$ e $7^{\circ}$ ano do ensino fundamental entre a faixa etária de 12 a 14 anos e 12 idosas que fazem parte de um grupo de dança, criado em um Centro de Convivência para idosos. Os acadêmicos de enfermagem solicitaram autorização da escola para a participação dos alunos e do Centro de Convivência para levar as idosas para uma apresentação de dança. O cenário para o desenvolvimento da ação foi o ginásio da escola, por ser um espaço maior, além de ter cobertura, boa iluminação e ventilação.

A experiência foi dividida em dois momentos principais: a) A ação apenas com os adolescentes no ginásio e; b) A ação entre as duas gerações, adolescentes e idosos. Para que a atividade pudesse envolver sua completude, os acadêmicos de enfermagem se dividiram em dois grupos para atender os adolescentes e as idosas. Utilizou-se como estratégia inicial não informar que haveria o encontro entre as duas gerações, sendo assim para as idosas foi explicado que seria uma festa de encerramento em uma escola e para os alunos que a atividade era sobre o processo do envelhecimento. As idosas ao chegarem na escola foram encaminhadas para uma sala e os adolescentes convidados para a atividade no ginásio. O objetivo foi permitir ao adolescente a oportunidade de expor livremente sua percepção sobre temáticas inerentes ao idoso. Posteriormente, o grupo de idosas foi convidado para ir até o ginásio para uma apresentação de dança. Apenas neste momento houve o encontro entre eles. Foram realizadas quatro atividades, a saber:

ATIVIDADE I: Dinâmica "Juntos e Misturados": foi desenvolvida apenas com os adolescentes, objetivando a troca de olhares sobre o envelhecimento. Utilizou-se a brincadeira do balão de ar contendo perguntas relacionadas a visão do adolescente em relação ao idoso quanto o uso de vestimentas curtas, sexualidade, uso de redes sociais, presença de senilidade, ser acompanhante nas atividades diárias e estatuto do idoso.

Todos os alunos se expressaram de alguma forma evidenciando a participação de todos. Esse resultado foi considerado positivo pelos acadêmicos de enfermagem, pois identificaram que é possível abordar sobre o envelhecimento com pessoas mais jovens, mas que a estratégia a ser realizada para conduzir esse processo deve ser dinâmico. Isso nos faz refletir sobre a importância das metodologias ativas utilizadas principalmente por ser um público mais jovem que se dispersa com facilidade. Quanto ao resultado desta dinâmica foi identificado atitudes positivas quanto ao respeito e a conduta em relação ao idoso.

ATIVIDADE II- Encontro entre os adolescentes e as idosas: os grupos iniciaram seu primeiro contato. Houve a apresentação pelas idosas de uma dança típica regional, o carimbó. Em seguida, os adolescentes se sentiram estimulados e entraram na dança por conta própria. A dança foi pensada como estratégia por três situações, primeiro porque o carimbó reflete a cultura do estado e por isso é conhecida por todos desde criança; a dança permite maior vínculo entre as pessoas; e porque a condução seria realizada por idosas.

REAS/EJCH | Vol. Sup. 23 | e413 | DOI: https://doi.org/10.25248/reas.e413.2019 Página 3 de $\mathbf{5}$ 
Buscamos observar como os adolescentes se comportariam neste momento. As expressões de susto foram percebidas ao verem as idosas entrando no ginásio com vestimentas típicas do carimbo e a realização da dança. Contudo, essas expressões foram dando espaço para expressões de alegria e movimentação do corpo. Analisando a dinâmica percebemos que obteve um resultado esperado que era de alcançar o vínculo entre as gerações.

ATIVIDADE III- Dinâmica "Juntos e Misturados": foi realizada novamente, mas apenas com as idosas, sendo assistidas pelos adolescentes. Esta estratégia teve como finalidade instigar a reflexão dos adolescentes sobre como os próprios idosos observam o envelhecimento. O resultado encontrado mostrou como as idosas se percebem atualmente. Sendo assim, se consideram ativas, capazes de exercerem suas atividades básicas, instrumentais e avançadas, autônomas, independentes e desenvolvem a sexualidade sem preconceitos ou medo. $O$ único destaque negativo foi quanto ao desrespeito que ainda perpassa a sociedade.

O momento de observação pelos adolescentes foi importante para que pudessem ter uma experiência com a realidade de ser idoso. $\mathrm{E}$ as idosas puderam exteriorizar sentimentos, ideias e costumes. Como acadêmicos de enfermagem entendemos a necessidade da troca de conhecimento e a importância de expressar sentimentos que muitas vezes não podem ser compartilhados por não ter apoio familiar e/ou social.

ATIVIDADE IV- $O$ balanço da roda entre duas gerações: as idosas e adolescentes foram convidados a entrar em uma roda, sendo que deveriam se abraçar, balançando os corpos devagar e sincronizados de um lado para o outro. Os acadêmicos de enfermagem iniciaram a condução da roda visando a reflexão sobre o encontro, destacando: a importância de se contar com o apoio não somente da família, mas também de amigos para conversar e estar nos momentos de "balanço" da vida. Ainda na roda, os acadêmicos ensinaram uma música intitulada "Levantar o braço", da Vovó Mafalda, com o final modificado para a dinâmica, sendo pedido que realizassem os movimentos de acordo com a letra.

Na finalização da atividade alguns participantes pediram a palavra para destacarem que foi a primeira vez que desenvolveram uma ação entre dois grupos e que ficaram satisfeitos. O principal desafio encontrado pelos acadêmicos na condução dessa ação educativa foi o receio da não aceitação dos adolescentes e das idosas, principalmente porque ao serem convidados não foi orientado quanto à participação de outro grupo, contudo, o feedback do grupo e o resultado de cada etapa permitiu entender o fortalecimento da proposta trazida pelos acadêmicos de enfermagem de que mesmo com a singularidade de cada grupo é possível a intergeracionalidade, pois são grupos que podem ter uma relação interpessoal e também troca de experiências e valores. Todos esses aspectos podem ser um suporte em potencial para a desconstrução de estereótipos da velhice.

\section{DISCUSSÃO}

A busca da aproximação entre gerações distintas deve ser estimulada e diante do contexto vivenciado atualmente quanto ao aumento do número de idosos na população total e o preconceito e estigma com este público é importante a busca de estratégias que visam aproximar a população idosa de outros grupos etários, como os adolescentes. O processo de intergeracionalidade evidencia que o preconceito tende a reduzir quando as pessoas desenvolvem a percepção de que os grupos a que pertencem e aqueles a que não pertencem são parte integrante da mesma comunidade (COELHO C, 2013).

Em uma pesquisa realizada por Pereira RF et al. (2014) com adolescentes de uma escola pública e privada sobre velhice identificou-se pontos distintos, pois a velhice foi considerada pelo adolescente como uma fase da vida que expressa autonomia, mas também muitas dificuldades devido a presença de patologias físicas e sociais.

O processo de senilidade não deve ser visto como uma consequência natural do envelhecimento, pois muitas pessoas, ainda adultos jovens, vivenciam limitações e patologias irreversíveis. Traçar uma sinonímia para o idoso como "uma pessoa doente" cria obstáculos na vivência entre gerações e aumenta o preconceito. Ainda se identifica na sociedade comportamentos inadequados em relação a uma pessoa baseada somente na sua idade, conhecido como comportamento idadista (SOUSA ACSN et al.,2014). 
Baseada nesta visão limitada, supracitada anteriormente, Martins SMF (2013) reforça o desenvolvimento das práticas de intergeracionalidade no combate à discriminação e estereótipos idadista e outros aspectos, pois é possível a aproximação entre as gerações visando a promoção do autoconceito, autoestima e bemestar.

A inclusão de programas intergeracionais devem ser destaques nas discussões frente ao envelhecimento. Para França LHFP et al. (2010) estes programas permitem a inserção do idoso dentro da sociedade ao aproximar de outras pessoas independentemente dos laços familiares. Em virtude dessa necessidade, justifica-se a escolha pelos acadêmicos de enfermagem em realizar com grupos que não se conheciam e ser realizado em um ambiente diferente.

Pesquisas científicas nacionais tem sido realizadas atualmente, mas a maioria destaca somente a importância da intergeracionalidade o envelhecimento A busca de projetos intervencionistas se torna necessária para que permita um contato com o público alvo, como a pesquisa desenvolvida no Paraná por Massi G et al. (2016) em que reuniu crianças, adolescentes e idosos por oito meses para o desenvolvimento de ações conjuntas sobre relatos pessoais envolvendo conflitos entre gerações no contexto familiar, conflitos intergeracionais no contexto corporativo e preconceitos intergeracionais.

\section{CONSIDERAÇÕES FINAIS}

A ação realizada permitiu identificar que a interação entre adolescentes e adultos é possível e que é uma estratégia que merece ser estudada na busca da desconstrução dos estereótipos da velhice. Não é fácil desenvolver ações entre gerações distintas, mas o primeiro passo precisa ser dado. O resultado da ação estimulou os acadêmicos de Enfermagem a ter um olhar mais crítico para a atual realidade. Destaca-se como limitação do estudo a falta de um acompanhamento por mais tempo dos grupos, contudo espera-se que os resultados encontrados possam estimular os pesquisadores, gestores, escolas, sociedade e os próprios profissionais da saúde na busca de estratégias e criação de projetos que permitam o fortalecimento da intergeracionalidade entre idosos e adolescentes.

\section{REFERÊNCIAS}

1. COELHO, C. Idades apartadas: pensar o idadismo e a intergeracionalidade. In: Pereira, J. et al. (Coord.) Animação Sociocultural, Gerontologia e Geriatria, A Intervenção Social, Cultural e Educativa na Terceira Idade. Intervenção - Associação para a promoção e divulgação cultural, Chaves, pp. 63-72, 2013.

2. DÁTILO G, CORDEIRO A. Envelhecimento humano, diferentes olhares. Cultura Acadêmica Editora, São Paulo, 2015.

3. DAWALIBI NH, et al. Envelhecimento e qualidade de vida: análise da produção científica da SciELO. Estudos de Psicologia, Campinas, 2013; 30(3): 393-403.

4. FRANÇA LHFP, et al. Programas intergeracionais: quão relevantes eles podem ser para a sociedade brasileira? Rev. Bras. Geriatr. Gerontol., 2010; 13(3):519-531.

5. MARTINS SMF. A Idade dos Afetos Avaliação de Necessidades de Contacto Intergeracional na Aldeia de Santa Isabel (Santa Casa da Misericórdia de Lisboa). Tese. (Mestrado em Psicologia). Escola de Ciências Sociais e Humanas Departamento de Psicologia Social e das Organizações. Lisboa, 2013; 167 p.

6. MIRANDA G, et al. O envelhecimento populacional brasileiro: desafios e consequências sociais atuais e futuras. Revista Brasileira de Geriatria e Gerontologia,2016; 19(3): 507-519.

7. MARTÍNEZ QUES ÁA. Ageismo y derechos humanos enel contexto Sanitario. Tese. (Doctorado em Derecho) - Universidad Nacional de Educación a Distância, España, 2015; 423 p.

8. MASSI G, et al. Impacto de atividades dialógicas intergeracionais na percepção de crianças, adolescentes e idosos. Rev. CEFAC, 2016;18(2):399-407.

9. SILVA DM da, et al. Dinâmica das relações familiares intergeracionais na ótica de idosos residentes no Município de Jequié (Bahia), Brasil. Ciência \& Saúde Coletiva [online], 2015; 20(7): 2183-2191.

10. SOUSA ACSN, et al. Alguns Apontamentos sobre o Idadismo: A posição de pessoas idosas diante desse agravo à sua subjetividade. Estudos interdisciplinares sobre o envelhecimento, Porto Alegre,2014; 19(3): 853-877.

11. TARALLO RS, et al. Atitudes de idosos e de profissionais em relação a trocas intergeracionais. Revista Brasileira de Geriatria e Gerontologia, Rio de Janeiro, 2017; 20(3): 423-431.

12. TORRES TL, et al. Estereótipos sociais do idoso para diferentes grupos etários. Psicologia: Teoria e Pesquisa, Brasília, 2016; 32(1): 209-218. 